\title{
Towards a Global Evaluation of Uncertainty for the Monitoring of Distribution Grids
}

\author{
Andrea Angioni*, Adriano Fioresi ${ }^{\dagger}$, Marco Pau*, Lorenzo Peretto ${ }^{\dagger}$, Ferdinanda Ponci*, Antonello Monti* \\ *Institute for Automation of Complex Power Systems, RWTH Aachen University, Aachen, Germany \\ $\dagger$ Dept. of Electrical, Electronic and Information Engineering, Alma Mater Studiorum University of Bologna, Bologna, Italy
}

\begin{abstract}
In electrical distribution networks many automation applications, such as voltage and power control, require to access the status of the system. Such information can be obtained by aggregating the heterogeneous measurements available in the grid and then applying state estimation algorithms. Different factors may have an impact on the estimation errors when the grid status evolves dynamically. The scope of this paper is to point out the different contributions affecting the global uncertainty of state estimation results when fast dynamics are present in the grid, for example due to fast variations of renewable energy sources generation or customers power consumption. The so-called "4 quadrants' method" is proposed to quantify and decompose such uncertainty components. The results of the 4 quadrants method may be useful in the process of design of the monitoring infrastructure.
\end{abstract}

\section{INTRODUCTION}

The increasing penetration of Distributed Generation (DG) and Distributed Energy Resources (DERs) connected to distribution systems calls for new distribution grid automation [1]. The main functionalities, such as power flow optimal control and lines protection, need an adequate monitoring of grid node voltages and line currents [2]. Furthermore, regulators are advancing new requirements on Power Quality (PQ) monitoring. This framework leads to the necessity of a performing monitoring system able to provide full and reliable observability of the grid conditions. Different factors characterize the monitoring system, like its computational efficiency, its robustness against data losses, etc. In this paper the focus is on the uncertainty features of the monitoring platform, with particular attention to the effects deriving by the dynamic phenomena usually present in distribution networks. Grid monitoring is fully developed in transmission systems [3]. The transmission buses are generally equipped with substation instrumentation and measurements are collected and exploited for real-time state estimation. The application of the same paradigm to distribution grids poses several challenges and clashes with some straight economic evaluations [4]. The main challenges are given by: 1 . the number of nodes of distribution grids, which is much larger than the one at transmission level; and 2. the poor coverage of measurement devices in distribution level. In the near future, the coverage of real-time measurements in distribution networks will be likely supported by Smart Meters (SMs) and Phasor Measurement Units (PMUs), in particular if low cost devices will become available in the market [5], [6]. All these heterogeneous measurements have different characteristics in terms of type of measured quantities (node voltage magnitude and phase angle, branch current magnitude and phase angle, active and reactive power flow and injection, energy injection); measurement time window (1 measurement every cycle, second or minute); measurement uncertainty. In a monitoring platform, State Estimation (SE) is responsible for aggregating such heterogeneous measurements and providing a coherent state of the system, with a given update rate. In presence of evolving dynamics of the state, different measurement time windows, the way measurements are aggregated and the placement of measurement devices may have a large impact on the accuracy of the estimator. Despite many literature contributions investigating the uncertainty of SE in steady state conditions [7]-[9], the uncertainty behaviour in dynamic conditions has been so far only partially evaluated in literature and on demonstration fields [10]. This knowledge gap has to be addressed in order to provide a correct information on the uncertainty of the monitored quantities to the upper level automation functions that leverage on SE results. In this way, possible harmful effects of this uncertainty can be reduced, avoiding wrong decisions that could jeopardize the operation of hte distribution grid [11]. This paper makes a step forward in this direction, presenting a method, named four quadrants' method, based on real-time tests to evaluate the global uncertainty of a monitoring platform, which relies on heterogeneous measurement devices, in presence of dynamic evolution of the state of the grid. A complete knowledge of the total uncertainty in presence of dynamic conditions may be useful in the process of design of the monitoring infrastructure. The four quadrants' method will be evaluated with two test cases based on high voltage and sun irradiation variations.

\section{UNCERTAINTIES IN MONITORING OF DISTRIBUTION}

A monitoring system uses the measurements available from the field and the knowledge of the grid model to obtain the status of the network in terms of voltages, currents and powers. The monitoring chain, shown in Fig. 1, is generally composed by: 1) sensors and transducers, 2) data acquisition (signal conditioning and analog to digital conversion), 3) measurement processors, 4) data concentrators, 5) SE algorithm. Some of the mentioned components may be integrated in a unique device. For instance SMs include sensors and transducers, analogue to digital converters and measurement processing units. PMUs or other Intelligent Electronic Devices (IEDs) may access sampled values from merging units or be directly plugged to the sensors and transducers. IEDs, PMUs and 
SMs usually send periodic (or event base) measurements through Local Area Networks (LANs) or Wide Area Networks (WANs) to data concentrators. Data concentrators then expose measurements adequately harmonized to the SE. The output of SE provides a picture of the operating conditions of the system and is used for simple monitoring activities and as input for automatic controllers or SCADA systems used by Distribution System Operators (DSOs).

\section{A. Contributions to the total uncertainty}

The contributions to the total uncertainty may come from any of the aforementioned components of the monitoring chain. In this paper three categories of measurement uncertainty will be considered, as presented in (1) and depicted in Fig. 2. The uncertainty due to errors of sensors, transducers, data acquisition and measurement processors, in case of steady state conditions, will be named $u_{s}$. The uncertainty arising in case of transient conditions is splitted into two contributions, named $u_{t}$ and $u_{d}$. The term $u_{t}$ is due to the delay of sensors, transducer and data acquisition in case of fast changes in their input. The term $u_{d}$ represents the uncertainty due to the methods used by measurement processor and data concentrator of handling sampled values and measurements, respectively. This includes the time window of measurements, the mathematical formula used to combined the sampled value in a measured value, the estimation time window and the method used by the data concentrator to harmonize data from non-synchronized measurement devices or measurement devices with different time windows. Such elements will have a significant uncertainty contribution only when the grid conditions are evolving during transient conditions. More details on $u_{s}, u_{d}$ and $u_{t}$ are given in subsection II-B.

$$
u_{\text {meas }}=\sqrt{u_{s}^{2}+u_{t}^{2}+u_{d}^{2}}
$$

The overall uncertainty of the monitoring chain, $u_{\text {meas }}$, in this work, represents the uncertainty that affects the input signal of the SE. The uncertainty of the estimated state, $u_{\text {state }}$, is still to be determined as a function of the measurement uncertainty $u_{\text {meas }}$. As known from the literature (see for example [8], [12]), if the state estimation is calculated with traditional Weighted Least Squares approaches, its uncertainty can be found as:

$$
\mathbf{R}_{x}=\left[\mathbf{H}^{T} \mathbf{R}_{m}^{-1} \mathbf{H}\right]^{-1}
$$

where $\mathbf{R}_{x}$ is the covariance matrix of the estimated state vector, $\mathbf{R}_{m}$ is the measurement covariance matrix and $\mathbf{H}$ is the Jacobian matrix of the system. If the different components of the measurement uncertainty are independent, then the covariance matrix $\mathbf{R}_{m}$ can be decomposed in the sum of three terms, $\mathbf{R}_{m s}, \mathbf{R}_{m t}$ and $\mathbf{R}_{m d}$ (associated to $u_{s}, u_{t}$ and $u_{d}$, respectively). Therefore, also the estimation covariance matrix $\mathbf{R}_{x}$ can be then decomposed in three contributions $\mathbf{R}_{x s}$, $\mathbf{R}_{x t}$ and $\mathbf{R}_{x d}$ associated to the same terms. Thus, all the error components can be directly mapped as uncertainties of the SE

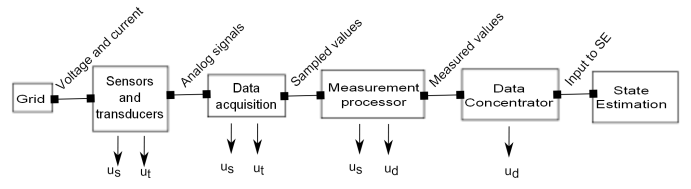

Fig. 1. Measurement chain and contribution to total uncertainty

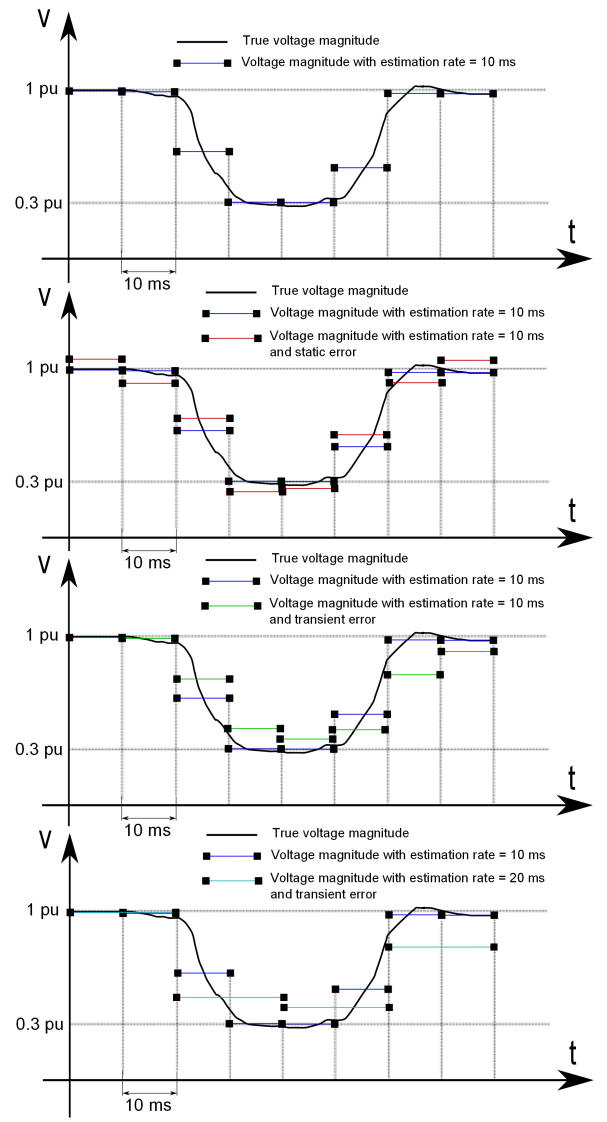

Fig. 2. Error components for voltage magnitude monitoring

results by applying a proper scaling by means of the Jacobian of the system.

\section{B. Characterization of the measurement uncertainties}

Sensors and transducers, as well as data acquisition systems, contribute to $u_{s}$ and $u_{t}$. The level of uncertainty may be calculated with calibrators and the methodologies described in standards (for instance IEC 61869-6 and IEC 61869-10). The sampled values, may be time tagged thanks to available time reference resources (for instance GPS units). Errors in the time tag may be accounted for in the term $u_{t}$. Some devices, as SMs, may not have time reference available, therefore the time tag is reconstructed at the data concentrator. In this case the contribution to $u_{t}$ depends on the communication infrastructure latency. Instead, measurement processing may affect the term $u_{s}$ and $u_{d}$. Methodologies for the evaluation of the total uncertainty brought by measurement processors are available, for instance in the standard C37.118.1 for PMUs, even though 
the two contributions, $u_{s}$ and $u_{d}$, are not clearly separated. Moreover, the time window of the measurement device may impact the $u_{d}$. For instance, a state estimator based on SM data updated every 30 seconds will have smaller $u_{d}$ than one based on SM data updated every 15 minutes. The data concentrator may receive measurements from devices with different time windows. Even when this is not the case, measurements may be referring to different time windows. The data concentrator, realize the so-called "data harmonization function", which provides a single input value for each electrical quantity to the SE. Different scenarios of data harmonization will have different impacts on $u_{d}$.

\section{Methodologies for the evaluation of $u_{d}$}

The quantification of $u_{d}$ would require assuming a reference method for measurement processing and a reference measurement and estimation rate. In the following, the results achievable calculating the phasors of voltage and current through the DFT, with a time windows of $20 \mathrm{~ms}$, updated every $10 \mathrm{~ms}$, will be used as base reference for the evaluation of $u_{d}$ in the other cases. In the test scenarios in this paper, SE and measurement time windows will be considered as an integer multiple of $10 \mathrm{~ms}$, as shown in (3):

$$
T_{w}=N \cdot 10[\mathrm{~ms}]
$$

The Root Mean Square Error (RMSE) index in (4) will be then used to evaluate the uncertainty component $u_{d}$ :

$$
R M S E_{k}=\sqrt{\frac{1}{N} \sum_{n=1}^{N}\left(\bar{x}_{k}-x_{k, n}\right)^{2}}
$$

where $R M S E_{k}$ is the RMSE calculated for the generic quantity $k, \bar{x}_{k}$ is the reference true value of the quantity $k$ calculated through the samples of the true quantity over the considered $20 \mathrm{~ms}$ time window, and $x_{k, n}$ is the $n$-th measurement of $k$. Differently from the case of $u_{s}$ and $u_{t}$, which may be evaluated on a single node (or branch for the current) trough dedicated calibrators, the term $u_{d}$ is influenced by the particular power system to be monitored and, in particular, by the disturbances that are likely to happen (fast and strong sun irradiation or load consumption variations). The magnitude and time duration of the voltage and current oscillations depend on the magnitude and time duration of the perturbations applied and the response of the power system. The type of perturbations considered in this paper are variation of voltage RMS, variations of solar radiation, variations of users' load or storage systems' power injections. The evaluation on $u_{d}$ is valid on a particular system, or more in details, for the response of the system to a particular set of the aforementioned types of perturbations.

\section{FOUR QUADRANTS' METHOD}

In the following, the methodology used to evaluate the previously mentioned uncertainty components is referred as to "Four quadrants' method". It includes a set of four tests intended to determine the 3 contributions of uncertainty $\left(u_{s}\right.$, $u_{t}, u_{d}$ ) in a monitoring platform.

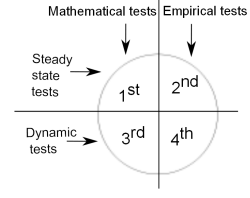

Fig. 3. Measurement chain and contribution to total uncertainty

\section{A. Four quadrants' testing procedure}

The first two quadrants are used to identify the $u_{s}$ contribution. The $3^{\text {rd }}$ and $4^{\text {th }}$ quadrants are intended to extract the combination of $u_{t}$ and $u_{d}$ in case of dynamic scenarios in the grid. The $1^{\text {st }}$ and the $3^{r d}$ quadrants calculate the measurement uncertainty, then the estimated state uncertainty $u_{\text {state }}$ is calculated through equation (2). Being based on the calculation of $u_{\text {state }}$ as a mathematical function of $u_{\text {meas }}$, the $1^{\text {st }}$ and the $3^{\text {rd }}$ quadrants are named also "mathematical" approaches. The $2^{\text {nd }}$ and the $4^{\text {th }}$ quadrant calculate instead directly $u_{\text {state }}$ through statistical tests and for this reason are also called "empirical". The structure of the four quadrants' method is presented in fig. (3). As demonstrated in [8], in steady state conditions the $u_{\text {state }}$ calculated with the mathematical and empirical approaches are the same. Section V demonstrates that the same conclusions also hold for the dynamic scenarios.

1) The $1^{\text {st }}$ quadrant, static mathematical approach: In the $1^{\text {st }}$ quadrant, the steady state measurement uncertainty is propagated to the state uncertainty using (2). The uncertainty $u_{s}$ may be obtained from data sheets or from metrological evaluation in laboratory environment. In this paper, the measurement infrastructure used for the tests includes some prototypes not yet metrologically characterized. Hence, tests were performed to have a preliminary characterization of these devices by using as data reference the quantities produced by a real-time digital simulator. This evaluation does not satisfy standard metrological requirements, but is sufficiently accurate to demonstrate the validity of the 4 quadrants' method in this work. The voltage measurement chain was fed with input values in steady state equal to $0.8,1$ and $1.2 \mathrm{pu}$; whereas the current measurement chain was fed with input values in steady state equal to $0.05,0.2,1.0$ and $1.2 \mathrm{pu}$, following the requirements of the standards IEC 61869-6 and IEC 61869-10 on low power voltage and current transformers, respectively. The measurement uncertainty was then evaluated as RMSE, obtained comparing the measurement output of the devices with the true values. This way the term $u_{s}$ is calculated.

2) The $2^{\text {nd }}$ quadrant, static empirical approach: In the $2^{\text {nd }}$ quadrant, the SE uncertainty is evaluated by comparing the estimation output with the true state given by digital simulation. The $2^{\text {nd }}$ quadrant considers, as the $1^{\text {st }}$ quadrant, the grid in steady state conditions. In the following tests voltage state conditions equal to $0.8,1$ and $1.2 \mathrm{pu}$ were reproduced in order to resemble the standard IEC 61869-6 and to extend the methodology applied on measurement devices to the overall monitoring platform. SE based on these values is performed and the output are stored for the RMSE calculation, 
which is here used as indicator of the total uncertainty of the estimated state. Also in this case, as the used signals are in steady state, terms $u_{t}$ and $u_{d}$ are zero and the component $u_{s}$ is calculated.

3) The $3^{\text {rd }}$ quadrant, dynamic mathematical approach: The $3^{\text {rd }}$ quadrant considers the dynamic scenarios and calculates the SE uncertainty based on the one of the measurements exploiting (2). Among the many possibilities to recreate grid dynamic scenarios, the authors selected two of the four meaningful dynamic cases, representing slow and fast variations of High Voltage (HV), sun irradiation and customer loads.

4) The $4^{\text {th }}$ quadrant, dynamic empirical approach: The $4^{\text {th }}$ quadrant considers the dynamic scenarios as the ones presented in the $3^{\text {rd }}$ quadrant, but it measures directly the RMSE of the estimated state.

\section{B. evaluation and comparison of results of the 4 quadrants}

In case the $1^{\text {st }}$ quadrant may be calculated exploiting laboratory calibration tools or real time digital simulators it is assumed that the $1^{\text {st }}$ and the $2^{\text {nd }}$ quadrant will provide equal or similar results. Otherwise, the $1^{\text {st }}$ quadrant may be applied, exploiting the indications of sensors, transducer, data acquisition and measurement processors data sheets, providing a proper representation of measurement and state uncertainty due to the combination of $u_{s}$ and $u_{t}$. Evaluation of $3^{r d}$ and $4^{\text {th }}$ quadrants require the availability of real time digital simulators in laboratory environment, whit which to fed the monitoring chain. $3^{r d}$ and $4^{\text {th }}$ quadrants should provide equal or similar results of the total measurement and state uncertainty. The combined uncertainty includes the contribution due to $u_{s}, u_{t}$ and $u_{d}$. If the numerical samples of the simulation environment may be stored with an accurate time tag, it may be possible to feed the measurement chain with measurement input without any contribution of $u_{s}$ and $u_{t}$. Summarizing, the $1^{\text {st }}$ (or equivalently, the $2^{\text {nd }}$ ) quadrants provide the $u_{s}$; by comparing the results of the $3^{\text {rd }}$ (or equivalently, the $4^{\text {th }}$ ) and the $1^{\text {st }}$ (or equivalently, the $2^{\text {nd }}$ ) quadrants it is possible to extract the combination of $u_{t}$ and $u_{d}$; exploiting numerical samples from the simulation environment in the $3^{r d}$ (or equivalently, the $4^{\text {th }}$ quadrant) it is possible to extract the $u_{t}$.

\section{TEST SETUP}

The test setup used to demonstrate the 4 quadrants method is composed by the Real Time Digital Simulator (RTDS) which provide the values of currents and voltage in the distribution system modeled. The simulation quantities are provided in numerical format, resembling Smart Meters (SM) data, or in analogue format, to sensors and transducers feeding commercial PMUs. A computer acts as data concentrator for both SM and PMU data. The measurement data are stored a database and periodically accessed by the state estimator. A distribution system of 6 electrical nodes and 5 branches has been modelled in RTDS. The topology is presented in Fig. 4. The slack bus, represents the secondary side of the HV/MV transformer. The passive power injection at the nodes are

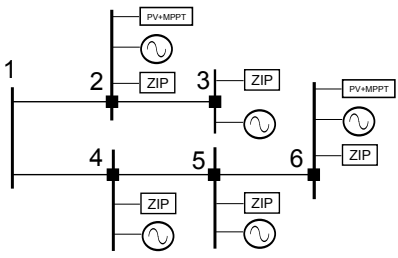

Fig. 4. Topology of tested power distribution system

composed by the sum of ZIP models and asynchronous motors as presented in [10]. The active power injection is represented by a mathematical model of several PV cells' strings and the MPPT based inverter as in [10].

\section{A. Sensors and measurement devices}

The voltage and current signals generated in RTDS are provided in numerical format trough the DNP3 protocol by the GTNET output card, thus resembling the behaviour of Smart Meters (SMs) or as voltage analog value, by the GTAO output card. The GTAO card includes 12 sixteen bit analogue output channels. It can provide output signals between $10 \mathrm{~V}$ peak. Oversampling is performed by the GTAO card and provides oversampled output every $1 \mathrm{~s}$. The GTNET produce numrical outputs with metrological features comparable to the one of the GTAO. Voltage sensors and current transducers, built with the combination of commercial converters, from ALTEA manufacturer, and labortory prototypes, are connected between the analog output of the RTDS and some commercial PMUs. The prototype amplifier, has been built on a Printed Circuit Board (PCB) in order to adapt the output of the RTDSGTAO (+-10V) to the input of the ALTEA converters (+$1 \mathrm{~V})$.The device may be categorized in class 0.5 , even tough the accuracy, was calculated using the metodology suggested in IEC 61869-7, but without certified calibrators. The ALTEA converters bring an input voltage in the range $+-1 \mathrm{~V}$ to the range $100 /$ sqrt(3) $\mathrm{V}$ for the voltage channels and $+-1 \mathrm{~A}$ for the current channel. They provide significant performances even during severe transients, and an overall good accuracy in term of ratio and phase error (class 0.5). The PMU generates synchrophasors at a rate that can be user-configured, following the IEEE C37.118 data format.

\section{B. Data concentrator and state estimator}

The synchrophasors and meter data are delivered trough a laboratory ethernet network, with negligible delay and packet losses, to the Data Concentrator. A computer acts as data concentrator for both SM and PMU data. The SM concentrator is resembled by the software KepServerEX, which permits to implement DNP3 masters, and withodrow measurements at a rate up to teens of ms. The Phasor Data concentrator (PDC) is resembled by the software OpenPDC. The measurement PMU and SM data are stored, respectively, in a MySQL and a PSQL database. PMU data are stored with the time tag provided by the device itself (the maximum error in time is below $10^{-6} s$ ), whereas the SM data are linked with the time read by the database from a NTP server (the maximum 
error in time is below $1 \mathrm{~ms}$ ) when the data is written. The state estimator estimates the voltage nodes phasors using the Weighted Least Square (WLS) Method [paper ref]. The weights are the inverses of the squares of the measurement uncertainties. Such estimator has been characterized in steady state conditions in [7], [9].

\section{Method for RMSE evaluation}

RTDS may store the simulation data, in order to be used a posteriori, for evaluation of the RMSE. Samples are stored every $2 \mathrm{~ms}$ and time tagged with a time source provided through the GTSYNC card, which is fed by the same GPS units that is used for PMUs. Applying the DFT, it is possible to calculate the corrisponding synchrophasor to be used as reference for the evaluation of the RMSE in the test scenarios.

\section{TEst Results}

Test result in this chapter are intended to: a. show the presence of $u_{d}$ and its contribution versus $u_{\text {meas }}$; b. show the effectiveness of the 4 quadrants method to calculate $u_{t}, u_{d}$ and $u_{\text {meas }}$. The application on a realistic distribution network also allow to draw generic conclusions on the features of the monitoring chain that have the largest impact on the total state uncertainty.

\section{A. Static case, Comparing outputs of $1^{\text {st }}$ and $2^{\text {nd }}$ quadrant}

The uncertainty here evaluated is the $u_{s}$ of the monitoring platform for a given power system, as indicated in section IV, measurement placement configuration, that is 1 PMU placed at bus 5 and pseudo measurements in the other buses. PMU is assumed by the state estimator, to have maximum error of bus voltage magnitude equal to $0.33 \%$ and phase angle equal to $0.09 \mathrm{crad}$ and maximum error of branch current magnitude equal to $0.51 \%$ and phase angle equal to $0.39 \mathrm{crad}$; pseudo measurements are expected to have $50 \%$ of maximum error of both active and reactive power readings. As presented in table I, the two quadrants give comparable results. Both of them have been evaluated with nominal voltage in the range $0.8,1$ and $1.2 \mathrm{pu}$, each one with 200 observations of 10 voltage and current signal cycles. In the $3^{\text {rd }}$ row the results are shown for the $2^{\text {nd }}$ quadrant, when the voltage in the grid is in steady state and close to $1 \mathrm{pu}$; it can be noticed that the term $u_{s}$ is significantly lower than the other two cases. The results of the $3^{\text {rd }}$ row will be exploited when comparing the first two quadrants with the second two in order to extract $u_{d}$ and $u_{t}$.

B. Dynamic case, comparing outputs of $3^{\text {rd }}$ and $4^{\text {th }}$ quadrant, fast voltage variation

One of the transient scenarios considers the effect of a voltage disturbance in the HV side of the primary transformer. The voltage at the slack bus instantly goes from 1 pu to $0.3 \mathrm{pu}$, and stay constantly equal to $0.3 \mathrm{pu}$ for 0.5 seconds, after that it rises, instantly, back to $1 \mathrm{pu}$. Such disturbance is propagated in the distribution network, yielding to variations in the current injection and consequently further oscillations in the voltage drops. The PMU is placed in bus 5, and has uncertainty as
TABLE I

UNCERTAINTY OF VOLTAGE PHASOR ESTIMATION

\begin{tabular}{|c|c|c|c|c|c|c|}
\hline index & 1 & 2 & 3 & 4 & 5 & 6 \\
\hline $\begin{array}{l}1^{\text {st }} \text { quadrant, volt- } \\
\text { age magnitude un- } \\
\text { certainty [\%] }\end{array}$ & 0.057 & 0.059 & 0.062 & 0.054 & 0.056 & 0.057 \\
\hline $\begin{array}{l}2^{n d} \text { quadrant, volt- } \\
\text { age magnitude un- } \\
\text { certainty [\%] }\end{array}$ & 0.049 & 0.049 & 0.050 & 0.049 & 0.050 & 0.050 \\
\hline $\begin{array}{l}2^{\text {nd }} \text { quadrant, volt- } \\
\text { age magnitude un- } \\
\text { certainty [\%] calcu- } \\
\text { lated with voltage } \\
\text { close to } 1 \mathrm{pu}\end{array}$ & 0.016 & 0.012 & 0.008 & 0.025 & 0.026 & 0.027 \\
\hline
\end{tabular}

described in the previous subsection V-A. It reports voltage and current synchrophasors 10 times per second. The SE is repeated 5 times per second. Each estimation window will have one synchrophasor available as entry to the state estimation. The observability is reached in the other nodes through pseudo-measurements, which have accuracy as in subsection V-A. Table II shows that the $3^{r d}$ and the $4^{\text {th }}$ quadrants give very similar results. Furthermore in the $3^{\text {rd }}$ row, the resulting uncertainty that is non-steady state (combination of $u_{d}$ and $u_{t}$ ), obtained subtracting under root square the squares of the 4 th and 2nd quadrant uncertainty results. It is possible to see from Fig. 5 that the contribution $u_{t}$, mainly due to the PCB prototype, built to adapt the voltage range of the RTDS to the ALTEA converters, is the main component to the total uncertainty. In fig 5 the plots of the estimated state versus the true state are shown.

\section{Dynamic case, sun irradiation variation}

The following transient scenarios considers the effect of the sun irradiation variation, similarly to the one reported during the solar eclipse of the 20th March 2015. The solar irradiation has been linearly reduced, in simulation environment, from $1000 \mathrm{~W} / \mathrm{m} 2$ to $250 \mathrm{~W} / \mathrm{m} 2$ in 1 minute and again raised up to $1000 \mathrm{~W} / \mathrm{m} 2$ in the next minute. The result in term of variation of current injection, propagates in the grid in terms of variation of voltage drop on the lines. The PMU is placed in bus 5, and has uncertainty as described in the previous subsection $\mathrm{V}-\mathrm{A}$. It reports voltage and current synchrophasors 10 times per second. The active and reactive power injection in the other nodes is read through smart meter measurements every 30 seconds. The SE assumes that the maximum error of smart

TABLE II

UNCERTAINTY OF VOLTAGE PHASOR ESTIMATION

\begin{tabular}{|l|c|c|c|c|c|c|}
\hline index & 1 & 2 & 3 & 4 & 5 & 6 \\
\hline \hline $\begin{array}{l}3^{\text {rd }} \text { quadrant, volt- } \\
\text { age magnitude un- } \\
\text { certainty [\%] }\end{array}$ & 0.252 & 0.252 & 0.252 & 0.252 & 0.252 & \\
\hline \hline $\begin{array}{l}4^{t h} \text { quadrant, volt- } \\
\text { age magnitude un- } \\
\text { certainty [\%] }\end{array}$ & 0.254 & 0.254 & 0.253 & 0.252 & 0.250 & 0.249 \\
\hline $\begin{array}{l}\text { non-steady state } \\
\text { voltage magnitude } \\
\text { uncertainty [\%] }\end{array}$ & 0.251 & 0.252 & 0.252 & 0.251 & 0.251 & 0.250 \\
\hline \hline
\end{tabular}




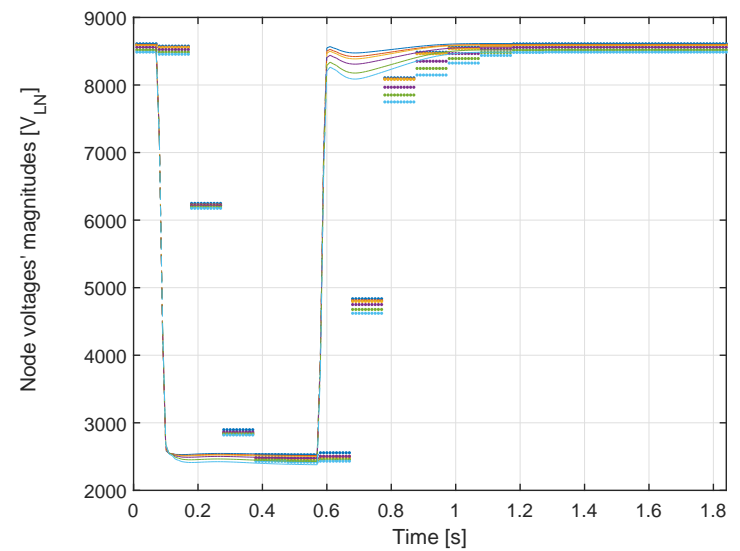

Fig. 5. Test setup

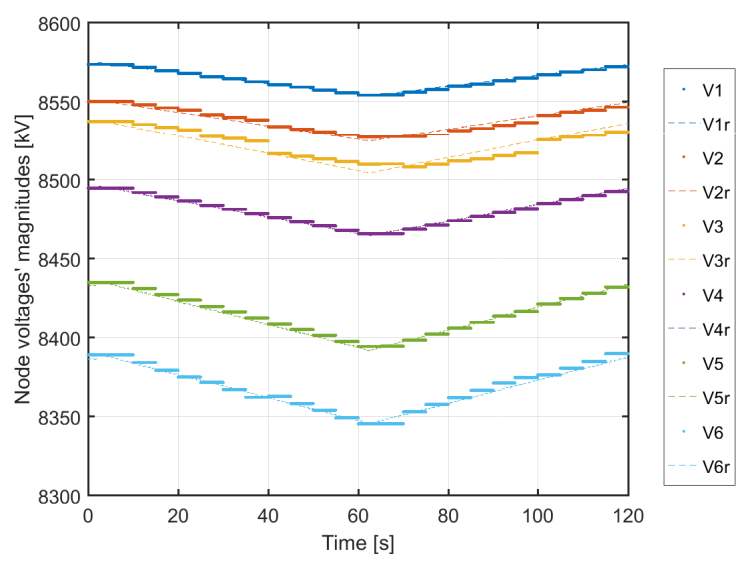

Fig. 6. Test setup meter data is $5 \%$. The state estimation is repeated 1 time every 5 seconds. The SE will exploit the last received phasor and the last updated SM data (even if it refers to older time windows). The observability condition is reached without the need of pseudo-measurements. Table III shows that the $4^{\text {th }}$ quadrant results (equivalent results are obtained with the $3^{\text {rd }}$ quadrant); furthermore, in the second row, the non-steady state uncertainty, obtained subtracting under root square the squares of the $4^{t h}$ and $2^{\text {nd }}$ quadrant uncertainty results, is presented. It can be noticed, that even in case of slow variations, due to sun irradiation changes, the contribution of dynamic uncertainty, this time, mainly due to $u_{d}$, is very large. In fig 6 the plots of the estimated state versus the true state are shown.

\section{CONCLUSION}

The method of the four quadrants has been presented as an effective methodology to coordinate laboratory demonstrations, based on laboratory calibrators or accurate real time simulators to characterize the main components of uncertainty in monitoring platform for distribution systems. It has been shown that realistic dynamic scenario may bring uncertainty components that are related to the feature of the power systems rather than the single device. Voltage oscillations and sun irradiation variations have been studied as test cases. An important impact is connected to the measurement and estimation time windows. The four quadrant methodology, may be further used for ad-hoc analysis of the total uncertainty in specific distribution network or for further characterizing

TABLE III

UNCERTAINTY OF VOLTAGE PHASOR ESTIMATION

\begin{tabular}{|l|c|c|c|c|c|c|}
\hline index & 1 & 2 & 3 & 4 & 5 & 6 \\
\hline \hline $\begin{array}{l}4^{t h} \text { quadrant, volt- } \\
\text { age magnitude un- } \\
\text { certainty [\%] }\end{array}$ & 0.025 & 0.056 & 0.132 & 0.019 & 0.030 & 0.028 \\
\hline $\begin{array}{l}\text { non-steady state } \\
\text { voltage magnitude } \\
\text { uncertainty [\%] }\end{array}$ & 0.02 & 0.055 & 0.130 & 0.017 & 0.015 & 0.004 \\
\hline \hline
\end{tabular}

each single component that contributes to the total uncertainty. For instance, the impact of choosing the last available entry or an average of the available entries by the state estimator when several measurements are available in a single estimation window. Or, the impact of having multiple measurements provided without time tag.

\section{REFERENCES}

[1] G. Heydt, "The next generation of power distribution systems," IEEE Trans. Smart Grid, vol. 1, no. 3, pp. 225-235, Dec. 2010.

[2] A. Meliopoulos, E. Polymeneas, Z. Tan, R. Huang, and D. Zhao, "Advanced distribution management system," IEEE Trans. Smart Grid, vol. 4, no. 4, pp. 2109-2117, Dec 2013.

[3] A. Monticelli, "Electric power system state estimation," Proc. IEEE, vol. 88, no. 2, pp. 262-282, Feb. 2000.

[4] D. Della Giustina, M. Pau, P. A. Pegoraro, F. Ponci, and S. Sulis, "Electrical distribution system state estimation: measurement issues and challenges," IEEE Instrumentation \& Measurement Magazine, vol. 17, no. 6, pp. 36-42, Dec. 2014.

[5] J. Liu, J. Tang, F. Ponci, A. Monti, C. Muscas, and P. Pegoraro, "Tradeoffs in PMU deployment for state estimation in active distribution grids," IEEE Trans. Smart Grid, vol. 3, no. 2, pp. 915-924, Jun. 2012.

[6] D. Macii, G. Barchi, and L. Schenato, "On the role of phasor measurement units for distribution system state estimation," in Environmental Energy and Structural Monitoring Systems (EESMS), 2014 IEEE Workshop on, Sept 2014, pp. 1-6.

[7] C. Muscas, S. Sulis, A. Angioni, F. Ponci, and A. Monti, "Impact of different uncertainty sources on a three-phase state estimator for distribution networks," IEEE Trans. Instrum. Meas., vol. 63, no. 9, pp. 2200-2209, Sept 2014.

[8] M. Pau, P. A. Pegoraro, S. Sulis, and C. Muscas, "Uncertainty sources affecting voltage profile in distribution system state estimation," in Instrumentation and Measurement Technology Conference (I2MTC), 2015 IEEE International, May 2015.

[9] C. Muscas, M. Pau, P. A. Pegoraro, and S. Sulis, "Uncertainty of voltage profile in PMU-based distribution system state estimation," IEEE Trans. Instrum. Meas., vol. 65, no. 5, pp. 988-998, May 2016.

[10] A. Angioni, I. Demchuk, F. Ponci, and A. Monti, "Specifying measurements' rates for monitoring of dynamic distribution grids," in 2016 IEEE International Instrumentation and Measurement Technology Conference Proceedings, May 2016, pp. 1-6.

[11] P. A. Pegoraro, F. Pilo, G. Pisano, S. Ruggeri, and S. Sulis, "Cosimulation of distribution active management and distribution state estimation to reduce harmful effects of inaccuracies," in PowerTech (POWERTECH), 2013 IEEE Grenoble, 2013, pp. 1-6.

[12] A. Gomez-Exposito, A. Abur, A. de la Villa Jaen, and C. Gomez-Quiles, "A multilevel state estimation paradigm for smart grids," Proc. IEEE, vol. 99, no. 6, pp. 952-976, June 2011. 